\title{
Role of conservative vs operative treatment for acute scaphoid fractures
}

\author{
Nihar Ranjan Mishra ${ }^{1}$, Satyajeet Ray ${ }^{2 *}$, R. C. Maharaj ${ }^{3}$, Subhranshu Sekhar Nayak ${ }^{4}$ \\ ${ }^{1}$ Post Graduate, ${ }^{2,3}$ Assistant Professor, ${ }^{4}$ Senior Resident, Dept. of Orthopaedics, SCB Medical College and Hospital, Cuttack, Odisha, India \\ *Corresponding Author: Satyajeet Ray \\ Email: drsatyajeetray@ rediffmail.com
}

\begin{abstract}
Scaphoid fracture is a difficult fracture to deal with. This tiny twisted bone, $80 \%$ covered with joint cartilage and lying angulated by $45^{\circ}$ in both planes deep in the wrist, has caused great frustrations among wrist surgeons. Diagnosis is difficult, classification is controversial $\&$ there is never ending debate on appropriate treatment protocol. Firstly, scaphoid fracture is prone to be missed in clinical assessment \& is usually neglected as simple wrist injuries. Adding to that, this notorious fracture is often missed in first x-ray immediately after injury. Second line of investigation is a matter of debate $\&$ varies from doctor to doctor $\&$ centre to centre. This does not end here. Once diagnosed, there is no clear-cut protocol for deciding appropriate treatment technique. There is controversy regarding whether to be managed conservatively or operatively. Both have their own pros \& cons. If managed conservatively, there is again no agreement in the literature as to the optimum position of immobilization (extension, ulnar deviation, neutral) or type of cast (thumb-spica, interphalangeal [IP] free/IP included, long arm/short arm). Last but the most nuisant are the complications. Scaphoid fracture is known for its complications like AVN, Non-union, arthritis owing to its peculiar blood supply, position in wrist \& shape. Management of these complications are again not free of confusions \& controversies.
\end{abstract}

In this study we have tried our best to address these dilemmas \& study this notorious bone in further detail.

Keywords: Acute scaphoid fracture, Treatment options.

\section{Introduction}

Scaphoid fracture is a difficult fracture to deal with. This tiny twisted bone, $80 \%$ covered with joint cartilage and lying angulated by $45^{\circ}$ in both planes deep in the wrist, has caused great frustrations among wrist surgeons. Diagnosis is difficult, classification is controversial \& there is never ending debate on appropriate treatment protocol. Firstly, scaphoid fracture is prone to be missed in clinical assessment $\&$ is usually neglected as simple wrist injuries. Adding to that, this notorious fracture is often missed in first $\mathrm{x}$-ray immediately after injury. Second line of investigation is a matter of debate $\&$ varies from doctor to doctor $\&$ centre to centre. This does not end here. Once diagnosed, there is no clear-cut protocol for deciding appropriate treatment technique. There is controversy regarding whether to be managed conservatively or operatively. Both have their own pros \& cons. If managed conservatively, there is again no agreement in the literature as to the optimum position of immobilization (extension, ulnar deviation, neutral) or type of cast (thumb-spica, interphalangeal [IP] free/IP included, long arm/short arm). Last but the most nuisant are the complications. Scaphoid fracture is known for its complications like AVN, Non-union, arthritis owing to its peculiar blood supply, position in wrist \& shape. Management of these complications are again not free of confusions \& controversies.

In this study we have tried our best to address these dilemmas \& study this notorious bone in further detail.

\section{Materials and Methods}

Over a period of two years (from DEC 2016 to December 2018) we performed a prospective cohort study of all patients presenting to our OPD/ Casualty with a suspected or confirmed injury to the scaphoid.

\section{Inclusion Criteria:}

1. All isolated acute scaphoid fractures $(<3$ weeks $)$ irrespective of the location.

2. All scaphoid fractures as a part of other acute injuries like peri-lunate instabilities.

The attending orthopaedic surgeon at the Emergency department or OPD examined all patients with posttraumatic radial sided wrist pain, who were suspected of having a scaphoid fracture. The clinical examinations consisted of three diagnostic tests: A. tenderness in the anatomical snuffbox (ASB); B. scaphoid tubercle tenderness (STT); and C. pain on longitudinal compression of the thumb (LTC). If any of these tests were positive, the patients were referred for a radiographic investigation of the wrist). The initial radiologic examination of the wrist included posteroanterior, lateral view, scaphoid view, oblique (45 deg. Pronation) view of the wrist. If the radiology reveals no fracture, wrist was immobilized with below elbow slab \& was instructed to review after 15 days. The same series of x-ray was repeated after 2 weeks. All patients were thoroughly counselled $\&$ instructed regarding participation in the study. If the patient accepted the invitation, they were enrolled in a prospective database, that included a patient questionnaire that sought information on sex, age, activity when the injury occurred (sport, traffic, work, or other), type of injury (fall, blow, or other), pathomechanism (extension, flexion, or other), and high-energy trauma (defined as a fall from $>1 \mathrm{~m}$ of height), nature of previous treatment received $\&$ past medical history etc.

Each patient was closely observed \& managed as per the recommended protocol. The pre-operative radiograph, range of motion, intra operative photographs, immediate post-operative radiographs, range of motion \& X-Ray in subsequent follow up were collected. 
In case of a clinically suspected scaphoid fracture without radiological signs of a fracture, early functional treatment was started using a below elbow slab. Patients with persistent clinical suspicion of a scaphoid fracture are repeated radiological evaluation after 2 weeks of the trauma to evaluate the current treatment strategy and to potentially adjust the treatment strategy as a result based on the radiographic findings. Displaced fracture is the one with $>2$ $\mathrm{mm}$ gap at fracture site. Several authors have reported that scaphoid fractures not visible on initial $\mathrm{x}$-ray films are "incomplete" or otherwise minor and will heal regardless of treatment. ${ }^{32,33,54}$ For practical purposes if a fracture is visible in all views immediately after injury, then it may be considered displaced. Otherwise a CT scan was done primarily to substantiate the clinical findings.

We advised for primary rigid fixation for all acute scaphoid fractures with consent of the patient. Those patients who were not agreed for operative procedure were managed with scaphoid cast (below elbow POP thumbspica cast in glass holding manner sparing the IP joint of thumb with thumb in palmar abduction and the wrist in neutral or slight extension).

Post operatively, a posterior POP cast was used to support the wrist for the first two weeks and then, after the removal of sutures, limb was immobilized for 4 weeks in case of fresh fractures $\&$ for 6 weeks in case of non-unions. After 6 weeks removable splint was applied for 6 weeks \& supervised physiotherapy was started gradually. Up to 12 weeks after surgery, patients were advised to avoid full loading of the wrist and to refrain from contact sports.

All patients were asked to attend for routine review at two and six weeks, three months, 6 month and one-year, additional visits being scheduled as required. Standard Scaphoid series radiographs were taken at each visit and a full clinical assessment was recorded. Modified MAYO Wrist score was used to assess functional out-come of individual patient after treatment. Patients who failed to attend for the six-month review were sent a questionnaire and encouraged to return for late clinical and radiological assessment. Radiological results were more rigorously defined: fractures were recorded as united, only if crosstrabeculation was present and the fracture line was no longer visible on any of the four standard views.

\section{Results}

A total of 37 cases of acute scaphoid fracture $(</=3$ weeks) were seen in a period of 2 years (Dec 2016 to Dec 2018).

The average age of presentation in our study was 36 years. The average delay in presentation was 1 week. This may be due to the fact that Scaphoid fracture is usually missed in initial standard AP \& Lateral X-Ray following an acute wrist injury or may be due to negligence of Patients in seeking medical consultation or both. Most common mode of injury was Road traffic accident. Other causes of injuries were sports injury, work place injuries, house hold injuries, assault injury.

Most common location of fracture was waist fracture (12 cases- 2 incomplete $\& 10$ complete) followed by distal oblique fracture (5 cases). 25 patients were managed conservatively \& 12 patients were treated with operative procedure (CRIF/ORIF WITN K-Wire / Herbert screw).

Mayo wrist score was calculated for all patients in follow up. Mean follow up period was 14 months. Out of all patients managed conservatively 7 patients had poor results $(<65), 2$ patient had fair results (65-79), 10 patients had good results (80-89), 6 patients had excellent results $(>/=90)$. In patients managed operatively poor results was obtained in 1 patient, fair result was obtained in 2 patients, good result was there in 4 cases \& excellent result was obtained in 5 cases.

All fractures managed by operative procedure (ORIF/per cut. Screw or k-wire fixation) united eventually. But there were 5 cases of non-union with conservative management.

Table 1

\begin{tabular}{|l|c|c|c|}
\hline & Right & Left & Total \\
\hline Male & $15(40 \%)$ & $13(35 \%)$ & $28(76 \%)$ \\
\hline Female & $6(16 \%)$ & $3(8 \%)$ & $9(24 \%)$ \\
\hline Total & $21(57 \%)$ & $16(43 \%)$ & 37 \\
\hline
\end{tabular}

Table 2

\begin{tabular}{|c|c|c|c|}
\hline Herbert Classification & Male & Female & Total \\
\hline A1 & 4 & 0 & 4 \\
\hline A2 & 2 & 2 & 4 \\
\hline B1 & 5 & 4 & 11 \\
\hline B2 & 10 & 1 & 6 \\
\hline B3 & 4 & 2 & 3 \\
\hline
\end{tabular}


Table 3

\begin{tabular}{|c|c|c|c|c|c|}
\hline & Poor & Fair & Good & Excellent & Total \\
\hline Conservative & 7 & 2 & 10 & 6 & 25 \\
\hline Operative & 1 & 2 & 4 & 5 & 12 \\
\hline Total & 8 & 4 & 14 & 11 & 37 \\
\hline
\end{tabular}

Table 4

\begin{tabular}{|c|c|c|c|}
\hline & Conservative & Operative & Total \\
\hline Union & 20 & 12 & 32 \\
\hline Non-union & 5 & 0 & 5 \\
\hline Total & 25 & 12 & 37 \\
\hline \% Age of non-union & $20 \%$ & $0 \%$ & \\
\hline
\end{tabular}

Case 1: 28 year old medical student presenting with displaced waist fracture managed conservatively
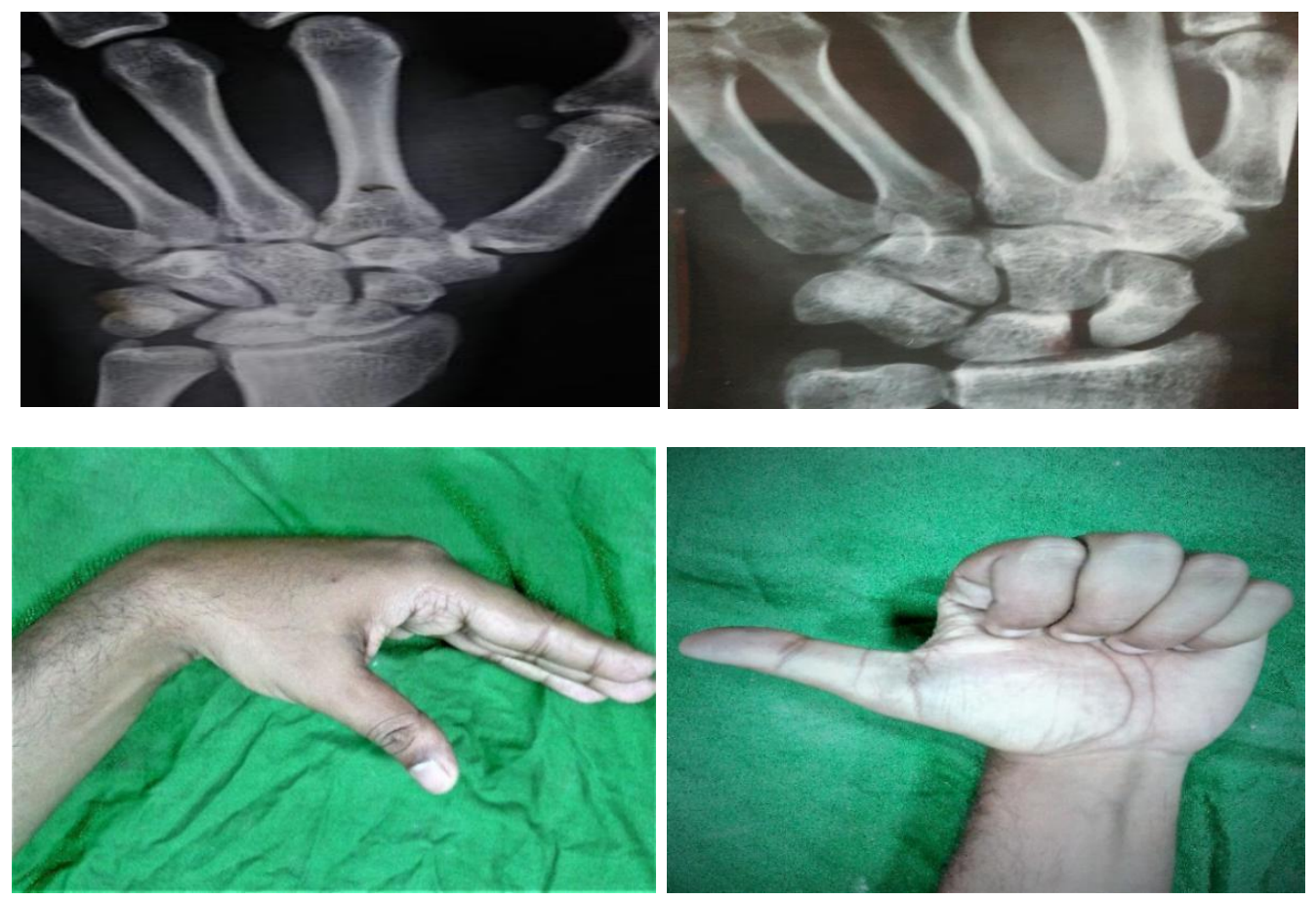

Case 2: 22 years old male dancer presenting after 1.5 months of RTA with SL ligament injury managed with per-cutaneous Herbert screw fixation:

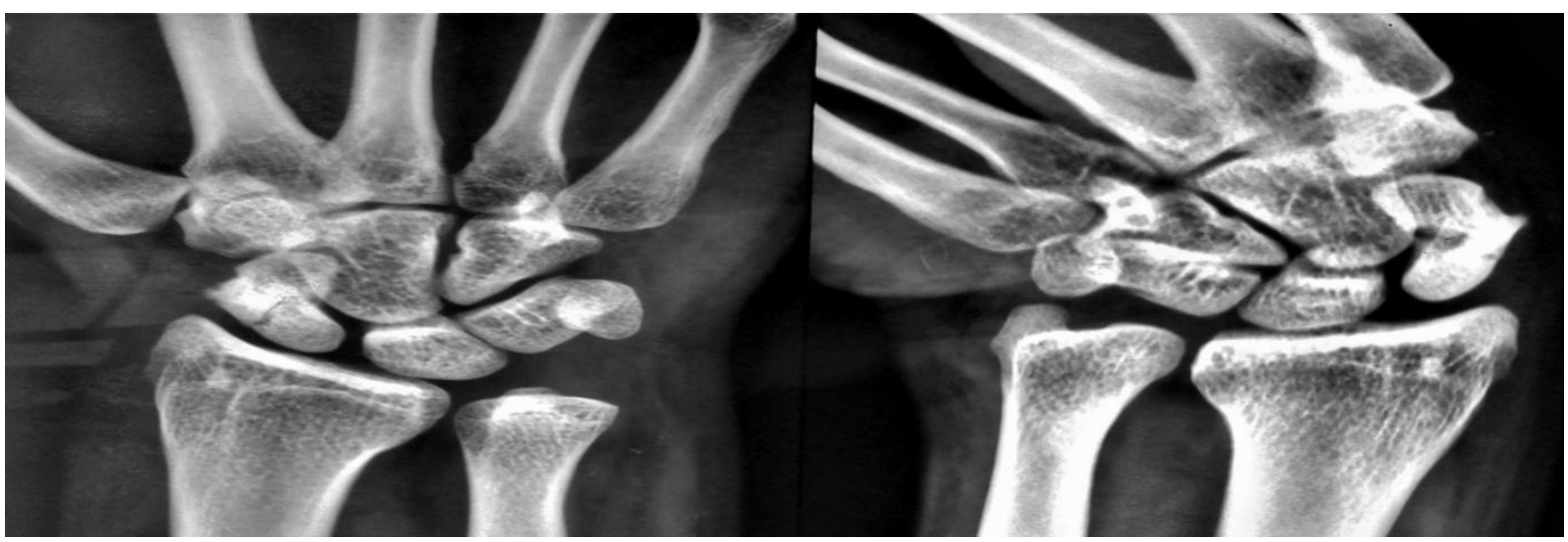



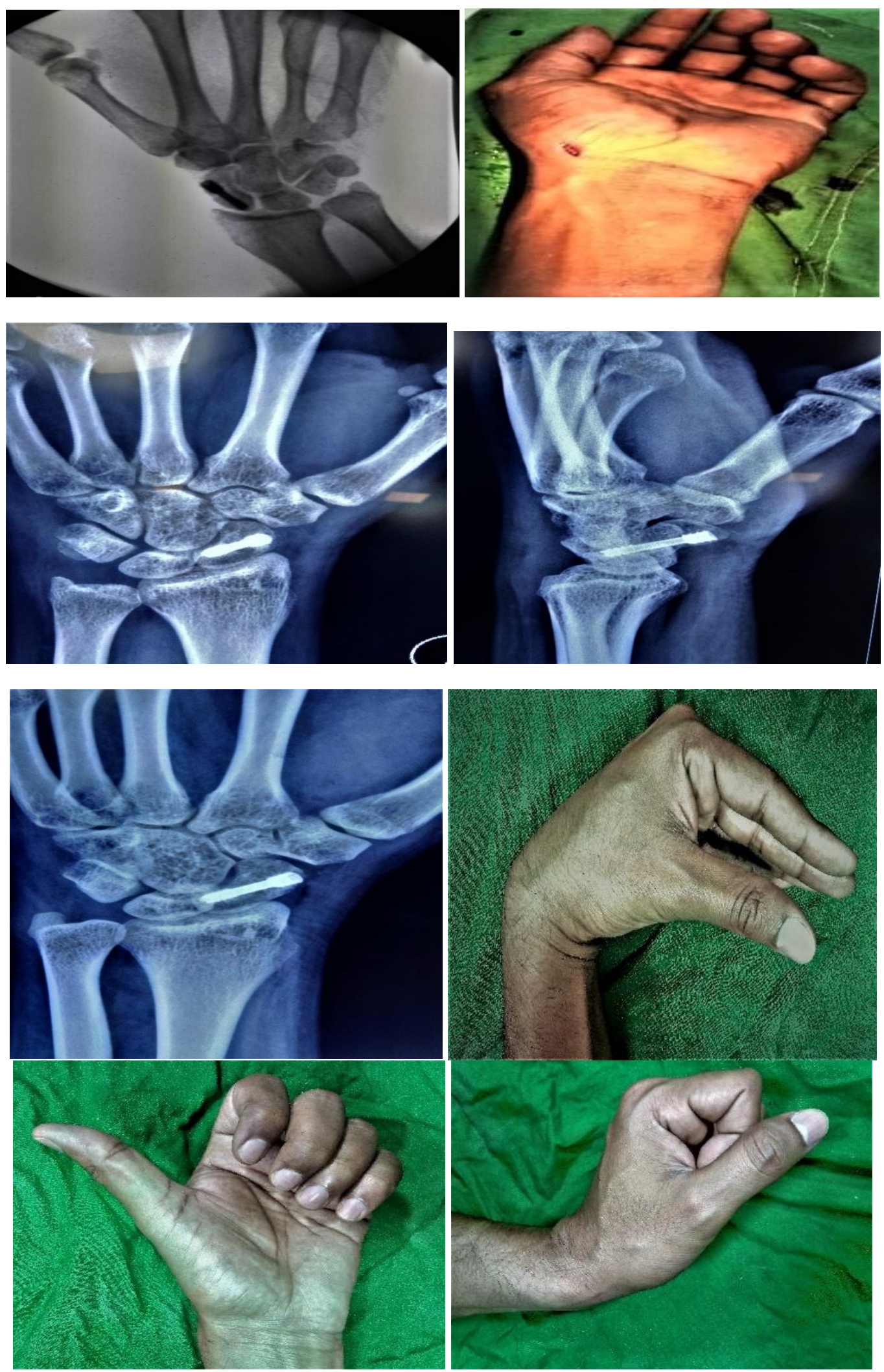
Case 3: 45 years male (doctor by profession) with distal oblique fracture with compound comminuted distal radius fracture treated with Open Reduction + Herbert screw fixation for scaphoid fracture \& External fixation \& K wire fixation for distal radius fracture, later developed extensor tendon contracture \& improved with supervised physiotherapy :
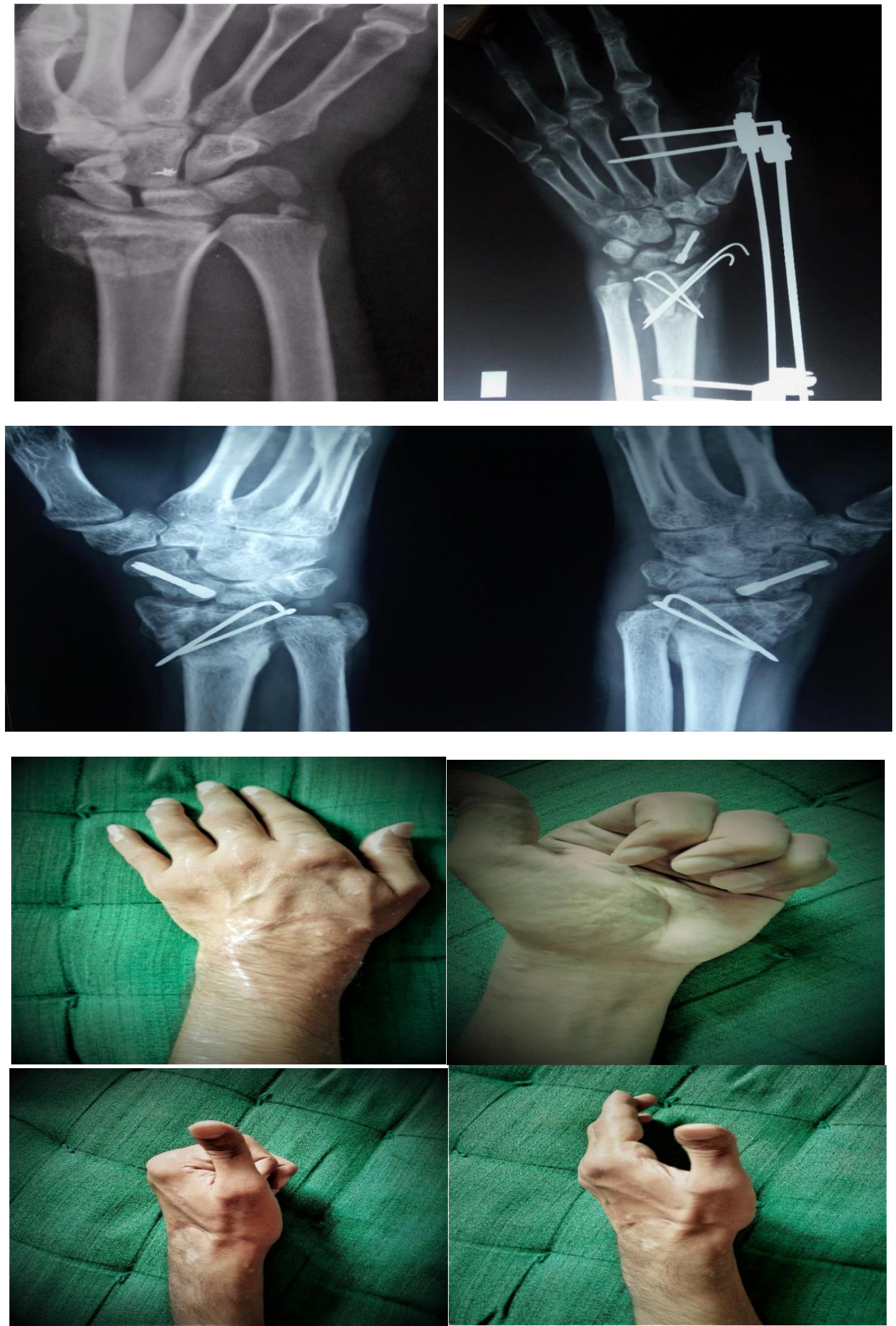


\section{Discussion}

Scaphoid fracture is a dilemma in orthopaedics owing to its delay in diagnosis (as in first presentation of pain in wrist joint, most often X-Ray of wrist in AP \& Lateral position are prescribed in which the fracture is often not seen), the controversial classification system and never-ending debate on appropriate treatment protocol.

The incidence of scaphoid fractures quoted in different literatures ranges from 1.47 to 121 per 100,000 of the population per year and is one of the key areas of discrepancy in the scaphoid fracture literature. This is likely due to the retrospective nature of many studies, low capture rates, small population sample sizes, the lack of a defined captive population, and the limitation of many databases in their ability to distinguish between a true and suspected fracture. The average age of presentation in our study was 36 years (16-86yrs). Most common age group involved was $30-39$ years $(31.5 \%)$. The trend tends to be decreased with extremities of age distribution.

In a review study conducted by Duckworth et al, ${ }^{1}$ Lowenergy falls from a standing height were most common $(40.4 \%)$ cause of scaphoid fracture. Contact sports comprised the next largest group $(n=35,23.5 \%)$, with football injuries being the most common $(n=24,68.5 \%)$. Major cause of injury in our study was Road traffic accident $(53 \%)$ followed by sports injury (16\%), work place injuries (14\%), house hold injuries (10\%), assault injury (7\%).

The classical clinical tests described for patients with post traumatic radial wrist pain anatomical snuffbox tenderness, scaphoid tubercle tenderness and pain at longitudinal compression of the thumb \& combinely, they have better specificity \& sensitivity $(97,98)$. Singh \& Dias ${ }^{2}$ described ASB tenderness, Effusion in USG, Scaphoid tubercle tenderness \& Scaphoid compression test for diagnosing scaphoid fracture. Chen ${ }^{3}$ has described the Scaphoid Compression Test, which is intended to discriminate scaphoid fracture from other causes of snuffbox tenderness. In his series of 52 traumatized wrists with snuffbox tenderness, he reports very high sensitivity and specificity of this test for scaphoid fracture. Others have not found such high specificity. The test is, at least, sensitive for fracture on the radial side of the wrist and thus provides an aid to diagnosis. The absence of scaphoid tubercle tenderness makes a diagnosis of scaphoid fracture unlikely. The absence of snuffbox and scaphoid tubercle tenderness virtually excludes a diagnosis of scaphoid fracture. We too allocated the patients depending on these initial three clinical tests at the time when the patient attended the Emergency Department/OPD.

Most common location of fracture reported by us was waist followed by distal oblique. Leslie \& Dickson ${ }^{4}$ also reported similar results.

First-line of radiologic investigation in patients with a suspected scaphoid fracture is wrist radiographs which are described to be good at ruling out a fracture (high specificity). ${ }^{5,6}$ All our patients had four scaphoid views \{PA, Lateral, Scaphoid view \& Oblique (45 deg. Pronation) view $\}$.
In addition to the standard PA and lateral radiographs used in the diagnosis of a scaphoid fracture, the additional views described in different literatures are the radial oblique view (proximal pole fractures), the ulnar oblique view (waist and tubercle fractures), and Ziter's view (waist fractures). Duckworth et $\mathrm{al}^{1}$ acknowledged that there is an intra- and interobserver error associated with interpreting the radiographs for the presence of a fracture, and classifying according to the Herbert classification, although this unavoidable.

Bohler et $\mathrm{al}^{8} \&$ Clay et $\mathrm{al}^{7}$ demonstrated that leaving the thumb free clearly allows patients greater hand function. Thus, we used a below elbow POP thumb-spica cast in glass holding manner sparing the IP joint of thumb with thumb in palmar abduction and the wrist in neutral or slight extension for patients managed conservatively.

Schubert opined that immobilization in the cast should continue until there are clinical and radiographic signs of bony union. These signs are absence of tenderness, appearance on x-ray films of bony trabeculae crossing the fracture line, a sclerotic band at the fracture site, or cortical continuity. The time varies directly with patients' age.

It is a common belief that the majority of scaphoid fractures will unite when treated in a cast for sufficient time. ${ }^{8-12}$ The average age of presentation in our study was 36 years. As most of the patients were active persons, the traditional treatment in a cast for several months may have inherent compliance problems, a substantial impact on daily living and a socioeconomic burden for society. Mink van der Molen et al. reported the time off work in carpal injuries to be 155 days in a cohort of 447 patients treated conservatively (98\%). Most patients were young men with manual work. ${ }^{13}$ The development of minimal invasive techniques in combination with an increasing demand from professional athletes of a quick functional recovery has evolved wrist surgeons globally toward offering percutaneous screw fixation of even un-displaced waist fractures to avoid plaster immobilization. ${ }^{14,15}$

Operative treatment for scaphoid fracture ensures two advantages. ${ }^{16-18}$ Firstly, patients will have an immediate fracture stabilization which is beneficial for healing and which allows for early return to normal activity. Secondly, preoperative assessment is insufficient for diagnosing the true nature of the fracture. Displacement and instability that are strongly associated with poor outcome, are most safely diagnosed in the operating theatre.

S. L. Filan, T. J. Herbert ${ }^{19}$ in 1996 stated in his review that apart from the one case of screw protrusion, they do not attribute any late non-unions to failure of fixation. Several of our patients sustained further injuries to their wrist within three months of surgery, but in none of these was outcome affected adversely. They therefore questioned the relevance of recent work which has compared the mechanical properties of different fixation devices in cadaver or foam model scaphoids. Their review has shown that a correctly inserted Herbert screw provides adequate fixation to allow early movement. While the same may be true for other devices, they believed that the challenge for the future is to 
secure adequate fixation with the least possible trauma to the bone and the surrounding joints and soft tissues.

Davis et $\mathrm{al}^{20}$ calculated that open reduction and internal fixation would be cost saving compared to casting from a societal perspective. They also found that the late consequences in the form of secondary arthritis were actually less in the operated group than in the conservatively treated group. In a Swiss study Fusseti et $\mathrm{al}^{21}$ reported that $34 \%$ of the individuals treated conservatively could resume work with the wrist immobilized in a cast. Furthermore, Papaloizos et $\mathrm{al}^{22}$ reported that operative treatment using a minimally invasive technique was initially more expensive than conservative treatment, but markedly decreased the work compensation cost and was less expensive overall compared to treatment in plaster.

In randomized clinical trials comparing the conservatively and surgically treated patients Bond and Saeden with co-workers found a significantly shorter period of sick leave in patients treated by percutaneous osteosynthesis. Differences in grip strength compared to the uninjured wrist between both groups were statistically insignificant with a better outcome in the surgically treated patients. Adolfson ${ }^{23}$ reported $13 \%$ mean loss of range of wrist motion in the conservatively treated group and $6 \%$ in the operated group.

Our results confirm that internal fixation leads to better functional results \& union of fracture than conservative treatment. We found more complain-free patients in the surgically treated group and less patients with resting pain and pain during sports and physical activities at the time of check-up which signifies a marked improvement in functional status with operative management.

The only disadvantage of Herbert screw fixation encountered by us was its technical difficulty. The operation requires skill and practice (Bunker et al 1987; Ford et al 1987; Smith et al 1991; Martini and Schiltenwolf 1993); poor surgery leads to poor results (Adams et al 1988). While doing per-cutaneous fixation, with the wrist in neutral position, the trapezium blocks the scaphoid bone. Thus, we prefer to give a small longitudinal incision $(\approx 1 \mathrm{~cm}$, or just long enough to accommodate the drill bit / guide wire) just distal to the scaphoid tubercle. The wrist is hyperextended and ulnarly deviated over a bump. This moves the trapezium dorsally away from the entrance point on the scaphoid bone. Another dis-advantage was un-availability of different size of screw specially for proximal $3^{\text {rd }}$ fracture for which 3.8 $\mathrm{mm}$ screw is larger. It would have been good to use a 2.7 $\mathrm{mm}$ screw. The average delay in presentation in this study was 1 week. Delay in seeking medical treatment is one of the risk factors for non-union. Also, patient compliance with cast immobilization is one of the important determinants of success of treatment procedure. We did not compare the time to union in the conservatively and surgically treated patients because, we did not perform CT examination routinely for all patients at the time of follow up evaluation. This was, along with a small sample, the main limitation of our study.

\section{Conclusion}

Based on our experience from this study we conclude that:

1. Anatomical snuffbox tenderness, scaphoid tubercle tenderness and pain at longitudinal compression of the thumb have high sensitivity to detect scaphoid fracture in wrist injury patients.

2. PA, Lateral, Scaphoid view \& Oblique (45 deg. Pronation) view are highly effective in detecting scaphoid fracture in patients with clinical suspicion. A repeat $\mathrm{x}$-ray is recommended after 2 weeks if fracture is missed but clinical examination is positive.

3. The choice of operative or nonoperative treatment must be individualized based on the discussion of pros and cons of treatment with the patient.

4. Early fixation promotes early mobilization \& early return to work, avoids development of complications in future \& improves over all functional outcome.

5. Non-operative treatment has good results in case of acute, non-displaced stable fractures through the scaphoid waist and in distal pole without other bony or ligamentous injury and for scaphoid fractures in children.

6. We recommend below elbow POP thumb-spica cast in glass holding manner sparing the IP joint of thumb with thumb in palmar abduction and the wrist in neutral or slight extension.

\section{Conflict of Interest: None.}

\section{References}

1. Andrew D. Duckworth, Paul J. Jenkins, Stuart A. Aitken, Nicholas D. Clement, Charles M. Court-Brown, MD, Margaret M. McQueen. Scaphoid fracture epidemiology. $J$ Trauma Volume 72, Number 2.

2. H.P. Singh, J.J. Dias. Focus on Scaphoid fractures. $\mathrm{Br}$ Editorial Soc Bone Joint Surg 2011.

3. Berger RA: The anatomy of the scaphoid. Hand Clin 2001;17(4):525-32.

4. Bain, G.I., Clinical Utilisation of Computed Tomography of the Scaphoid. Hand Surg 1999;4(1):3-9. Leslie, I.J. and R.A. Dickson, The fractured carpal scaphoid. Natural history and factorsinfluencing outcome. J Bone Joint Surg Br 1981. 63B(2): p. 225-30.

5. American College 10of Radiology. Appropriateness Criteria. Acute Hand and Wrist Trauma.2013 [cited1998]. Available at: http:// www.acr.org/ /media/ ACR/ Documents/ AppCriteria/ Diagnostic/ AcuteHandAndWristTrauma.pdf

6. Yin, Z.G., Zhang JB, Kan SL, Wang XG., Diagnostic accuracy of imaging modalitiesfor suspected scaphoid fractures: meta-analysis combined with latent class analysis. J Bone Joint Surg Br 2012;94(8):1077-85.

7. Clay NR, Dias JJ, Costigan PS, Gregg PJ, Barton NJ. Need the thumb be immobilized in scaphoid fractures? J Bone Joint Surg Br 1991;73B(5):828-32.

8. Bohler, L., E. Trojan, and H. Jahna, The results of treatment of 734 fresh, simple fractures of the scaphoid. J Hand Surg Br 2003;28(4):319-31.

9. Bain, G.I., Clinical Utilisation of Computed Tomography of the Scaphoid. Hand Surg 1999;4(1):3-9.

10. Leslie, I.J. and R.A. Dickson, The fractured carpal scaphoid. Natural history and factorsinfluencing outcome. J Bone Joint Surg Br 1981;63-B(2):225-30. 
11. Singh, H.P., Forward D, Davis TR, Dawson JS, Oni JA, Downing ND., Partial union of acute scaphoid fractures. $J$ Hand Surg Br 2005;30(5):440-45.

12. Geoghegan, J.M., Woodruff MJ, Bhatia R, Dawson JS, Kerslake RW, Downing ND, et al. Undisplaced scaphoid waist fractures: is 4 weeks' immobilisation in a belowelbowcast sufficient if a week 4 CT scan suggests fracture union? J Hand Surg Eur 2009.34(5):631-7.

13. Amirfeyz, R., Bebbington A, Downing ND, Oni JA, Davis TR. Displaced scaphoidwaist fractures: the use of a week 4 CT scan to predict the likelihood of union withnonoperative treatment. J Hand Surg Eur 2011;36(6):498-502.

14. Van der Molen, A.B., Groothoff JW, Visser GJ, Robinson $\mathrm{PH}$, Eisma WH. Time off work due to scaphoid fractures and other carpal injuries in The Netherlands in theperiod 1990 to 1993. J Hand Surg Br 1999;24(2):193-8.

15. Whipple, T.L., The role of arthroscopy in the treatment of wrist injuries in the athlete. Clin Sports Med 1998;17(3):62334.

16. Rettig, A.C., Athletic injuries of the wrist and hand. Part I: traumatic injuries of the wrist. Am J Sports Med 2003;31(6): 1038-48.

17. Cooney WP, 3rd. Scaphoid fractures: current treatments and techniques. Instr Course Lect 2003;52:197-208.

18. Haisman JM, Rohde RS and Weiland AJ. Acute fractures of the scaphoid. J Bone Joint Surg Am 2006;88:2750-8.

19. Haisman JM, Rohde RS and Weiland AJ. Acute fractures of the scaphoid. Instr Course Lect 2007;56:69-78.
20. S. L. FILAN, T. J. Herbert, Herbert Screw Fixation of scaphoid fractures. J Bone Joint Surg [Br] 1996;78-B:51929.

21. Davis EN, Chung KC, Kotsis SV, Lau FH and Vijan S. A cost/utility analysis of open reduction and internal fixation versus cast immobilization for acute nondisplaced mid-waist scaphoid fractures. Plast Reconstr Surg 2006;117:1223-35.

22. Fusetti C, Garavaglia G, Papaloizos MY, Wasserfallen JB, Büchler U and Nagy L. Direct and indirect costs in the conservative management of undisplacedscaphoid fractures. Eur J Ortop Surg Traumatol 2003;13:241-4.

23. Papaloizos MY, Fusetti C, Christen T, Nagy L and Wasserfallen JB. Minimallyinvasive fixation versus conservative treatment of undisplaced scaphoidfractures: a cost-effectiveness study. J Hand Surg [Br] 2004;29:116-9.

24. Barton NJ. Twenty questions about scaphoid fractures. $J$ Hand Surg [Br] 1992;17:289-310.

25. Adolfsson L, Lindau T, Arner M. Acutrak screw fixation versus cast immobilisation for undisplaced scaphoid waist fractures. J Hand Surg Br 2001;26(3):192-5.

How to cite this article: Mishra NR, Ray S, Maharaj RC, Nayak SS. Role of conservative vs operative treatment for acute scaphoid fractures. Indian J Orthop Rheumatol 2019;5(1):154-61. 